\title{
Electrochemical quartz crystal microbalance study of polyelectrolyte film growth under anodic conditions
}

\author{
Sara Nilsson, Fredrik Björefors and Nathaniel D. Robinson
}

\section{Linköping University Post Print}

\section{Tweet}

N.B.: When citing this work, cite the original article.

Original Publication:

Sara Nilsson, Fredrik Björefors and Nathaniel D. Robinson, Electrochemical quartz crystal microbalance study of polyelectrolyte film growth under anodic conditions, 2013, Applied Surface Science, (280), 783-790.

http://dx.doi.org/10.1016/j.apsusc.2013.05.062

Copyright: Elsevier

http://www.elsevier.com/

Postprint available at: Linköping University Electronic Press

http://urn.kb.se/resolve?urn=urn:nbn:se:liu:diva-95934 


\title{
Electrochemical quartz crystal microbalance study of polyelectrolyte film growth under anodic
} conditions

\author{
Sara Nilsson ${ }^{a}$, Fredrik Björefors ${ }^{b}$, Nathaniel D. Robinson ${ }^{a^{*}}$ \\ ${ }^{a}$ Transport and Separations Group, Department of Physics, Chemistry and Biology, Linköping University, \\ SE-581 83 Linköping, Sweden \\ ${ }^{b}$ Department of Chemistry - Ångström Laboratory, Uppsala University, Box 538, SE-751 21 Uppsala, \\ Sweden \\ * natro@ifm.liu.se; +4613282212
}

\begin{abstract}
Coating hard materials such as Pt with soft polymers like poly-L-lysine is a well-established technique for increasing electrode biocompatibility. We have combined quartz crystal microgravimetry with dissipation with electrochemistry (EQCM-D) to study the deposition of PLL onto Pt electrodes under anodic potentials. Our results confirm the change in film growth over time previously reported by others. However, the dissipation data suggest that, after the short initial phase of the process, the rigidity of the film increases with time, rather than decreasing, as previously proposed. In addition to
\end{abstract}


these results, we discuss how gas evolution from water electrolysis and Pt etching in electrolytes containing $\mathrm{Cl}^{-}$affect EQCM-D measurements, how to recognize these effects, and how to reduce them. Despite the challenges of using Pt as an anode in this system, we demonstrate that the various electrochemical processes can be understood and that PLL coatings can be successfully electrodeposited.

\title{
Keywords
}

Quartz Crystal Microgravimetry; QCM; Electrochemistry; Poly-L-lysine; Platinum electrode; Film Growth

\author{
Abbreviations \\ CA - chronoamperometry \\ CE - counter electrode \\ $\mathrm{CV}$ - cyclic voltammetry \\ EQCM - electrochemical quartz crystal microgravimetry (or microbalance) \\ ITO - indium tin oxide \\ LbL - layer by layer \\ OCP - open-circuit potential \\ OWLS - optical waveguide lightmode spectroscopy \\ QCM - quartz crystal microgravimetry (or microbalance) \\ QCM-D - quartz crystal microgravimetry with dissipation \\ PLL - poly-L-lysine \\ $\mathrm{RE}$ - reference electrode \\ WE - working electrode
}




\section{Introduction}

Since the first studies of Galvani, the use of electrodes for studying biological systems has offered both opportunities and challenges. Bare metal electrodes can be used for short-term experiments (like Galvani's), but long-term studies involving implanted electrodes, for example, require that the electrodes be biocompatible. Platinum is a common and robust electrode material for such applications [1]. However, as with nearly any metal surface, biological cells distance themselves from the electrode, reducing the effectiveness of electronic measurement and/or stimulation [2]. Coating such electrodes with softer materials, e.g. via a layer-by-layer (LbL) method, is an obvious possible solution [3]. Studies highlighting the absorption of polyelectrolytes at solid-liquid interfaces are numerous [4-8]. While considering variables such as ionic strength and solution $\mathrm{pH}$, most of these studies neglect the substrate's electric potential, which is the focus in this study [9].

Poly-L-lysine (PLL), a polyelectrolyte or a poly amino acid, is often one of the first materials that biologists turn to for coating "hard" materials. Despite its use with electrodes, the effect of applied potential on the growth rate and properties of PLL films remains a relatively unexplored area. Through electrostatic interactions, PLL also promotes neural cell adhesion [10]. Others have reported the electrochemical desorption of LbL films containing PLL with potential biological applications such as cell release $[3,11-13]$. In the case of LbL thin film assembly, the construction of films by alternately adsorbing positively- and negatively-charged polyelectrolytes has been investigated using optical waveguide lightmode spectroscopy (OWLS) or ellipsometry with indium tin oxide (ITO) as the substrate $[6,11,12,14,15]$ van Tassel et al. have also reported the continuous adsorption of a polyelectrolyte under an applied anodic potential [7] via a similar adsorption process for both strongly- and weaklycharged polymers $[8,16]$, and hypothesized that the film growth mechanism changes during the process [8], resulting in a different orientation of the polymer chains (secondary structure). The assembly of thin films of chitosan by anodic electrodeposition has also recently been reported [17]. At first glance, such 
observations are counterintuitive, as the positively-charged polymers should be electrophoretically attracted to the cathode, rather than the anode. However, the examples mentioned above, in which anodic deposition was studied, and the study presented here, each employ a flow cell, in which hydrodynamic convection transports the polyelectrolyte to the anodic interface.

QCM-D is a gravimetric technique well-suited for studying the growth of films [18], where small changes in the mass and/or rheological properties of a film grown on (and liquid adjacent to) a quartz crystal cause measurable changes in the crystal's resonance frequency and the rate at which vibrational energy is dissipated by the system. Furthermore, when the electrode on the top face of the quartz crystal is connected to a potentiostat as part of a 3-electrode electrochemical cell, common techniques such as cyclic voltammetry (CV) and chronoamperometry (CA) can be employed simultaneously, resulting in a technique often referred to as EQCM-D [19]. EQCM-D can be particularly useful for investigating polyelectrolyte films in which the secondary structure is hypothesized to vary, as changes in the orientation of the polymer chains will likely cause observable differences in the films' rheological properties.

In this project, we set out to study anodic PLL film growth on Pt electrodes in a flow cell via EQCM-D. The chemical mechanism of interest, the accelerated and film-limited deposition rate under anodic conditions, appears to be independent of the rate of proton generation via water electrolysis. The byproducts of that reaction, however, interfere both with the gravimetric measurement technique and the resulting film. Furthermore, we appear to remove part of the Pt during the process. Thus, besides verifying observations previously reported by others $[7,15]$, we report methods for identifying, and even dealing with, the challenges associated with gas evolution at and etching of a Pt electrode.

\section{Material and methods}

\subsection{Chemicals}


PLL (mol. wt. 70,000-150,000, Sigma Aldrich) was used in all experiments and diluted to $0.4 \mathrm{~g} / \mathrm{l}$ in HEPES buffer (4-(2-hydroxyethyl)-1-piperazineethanesulfonic acid) (Sigma Aldrich), adjusted to a pH of 7.4 by adding $\mathrm{HCl}(1 \mathrm{~mol} / \mathrm{l}$, from Fluka Analytical). To the HEPES buffer, $\mathrm{NaCl}$ (Sigma Aldrich) was added to achieve a concentration of $100 \mathrm{mM}$. Before use, the buffer was degassed with $\mathrm{N}_{2}$, ultrasonic treatment, and filtered. Deionized water $(18.2 \mathrm{M} \Omega \mathrm{cm})$, obtained from a Milli-Q system was used in the preparation of both the electrolyte and the stock solution of PLL. For washing the quartz crystals, a mixture of Milli-Q water $\mathrm{H}_{2} \mathrm{O}_{2}$ (30\%, Merck) and $\mathrm{NH}_{3}$ (25\%, Merck) (5:1:1 by volume), referred to as TL-1 in the text, was used. The crystals were submersed in the liquid at $80^{\circ} \mathrm{C}$ for $5 \mathrm{~min}$ and then rinsed 15 times in Milli-Q water prior to use.

\subsection{Quartz crystal microbalance}

The QCM used is an E4-system with an electrochemistry module (QEM 401, Q-Sense AB, Sweden). The sensors, i.e. the working electrode in the set-up, were Pt-coated ( $300 \mathrm{~nm}$ ) AT-cut quartz crystals (QSX 314, Q-Sense AB). After the washing procedure (see section 2.1) the Pt electrode was dried with $\mathrm{N}_{2}$, mounted in the electrochemistry module and the resonant frequencies for the odd overtones between the $1^{\text {st }}$ and $11^{\text {th }}$ harmonic of the crystal were obtained. In most cases, we report the change in frequency and dissipation of the $5^{\text {th }}$ and $7^{\text {th }}$ harmonics (approximately, 25 and $35 \mathrm{MHz}$ ), indicated as $\Delta \mathrm{f}_{5}$, $\Delta f_{7}$ and $\Delta D_{5}, \Delta D_{7}$ respectively. Here, the relative frequencies reported have been scaled by the harmonic number, simplifying the direct comparison of the various overtones. For example,

$$
\Delta f_{5}=\frac{\hat{f}_{5}-\hat{f}_{5}^{0}}{5}
$$

where $\hat{f}_{5}$ is the unscaled measured frequency of the $5^{\text {th }}$ overtone at any given time and $\hat{f}_{5}^{0}$ is the unscaled measured frequency of the $5^{\text {th }}$ harmonic at the beginning of the experiment. $\Delta f_{n}$ can, for measurements of rigid films under dry conditions (e.g. in air), be used to calculate the change in mass $\Delta \mathrm{m}$ on the crystal through the Sauerbrey relationship [20]. 


$$
\Delta m=-c \Delta f_{n}
$$

where $\mathrm{c}$ is a constant that depends on the crystal and sensor's dimensions, etc. In the case of the crystals used in this work (Q-Sense's quartz crystal), c has a value of $17.7 \mathrm{ng} \mathrm{Hz}^{-1} \mathrm{~cm}^{-2}$. For plastic and viscoelastic films, where the film absorbs acoustic energy, the Sauerbrey approximation is not appropriate and a more detailed model of the system should be used [4]. Hydrated polymer films such as PLL in an aqueous electrolyte often fall into one or both of these categories. Note that change in resonance frequency is of interest, we have chosen to define $\hat{f}_{n}^{0}$ such that $\Delta \mathrm{f}_{\mathrm{n}}$ is zero at a convenient time.

The dissipation, $D$ is defined as the ratio of the energy dissipated in one period of crystal oscillation to the energy stored in the oscillator [18]. In the Q-Sense instrument, this value is calculated from the time constant $\tau$ associated with the decay in the oscillation after the driving potential has been removed [21].

$$
D=\frac{1}{\pi f_{s} \tau}
$$

where $f_{S}$ is the resonance frequency of the crystal for the overtone being sampled. For a bare crystal in a semi-infinite viscous liquid, $\mathrm{D}$ should be proportional to the square root of the product of the viscosity and density of the fluid,

$$
D=2 \sqrt{\frac{f_{s}}{n \pi}} \frac{1}{v_{q} \rho_{q}} \sqrt{v_{1} \rho_{1}}
$$

where $n$ is the overtone integer, $f_{s}$ the fundamental resonance frequency, $v_{q}$ the viscosity of the quartz crystal $(3340 \mathrm{~m} / \mathrm{s}), \rho_{q}$ the density of the quartz sensor $\left(2260 \mathrm{~kg} / \mathrm{m}^{3}\right), v_{1}$ the viscosity of the liquid, and $\rho_{1}$ the liquid density [22].

$\Delta D_{5}$ is then the dissipation calculated based on the $5^{\text {th }}$ overtone, measured relative to the dissipation at the starting point of the measurement (where $\Delta D_{5}$ is defined as zero). For very rigid films (e.g. metals) in vacuum or gaseous environments, where the Sauerbrey approximation above is most applicable, the change in dissipation $\Delta \mathrm{D}$ with changes in the film thickness is negligibly small. However, 6 of 27 
in more viscous environments such as the aqueous electrolytes used in the work presented here, $\Delta D$ is sensitive to the density and viscosity of the liquid through (4). Viscoelastic films, such as the PLL film studied in this work, also cause $\Delta \mathrm{D}$ to increase.

We wish to note that the removal of Pt, described in section 3.4.2 also depends on the Pt sensors themselves. Several batches of Pt quartz crystal sensors were unusable, as they were quickly destroyed because the metal layers detached from the quartz crystal when a potential was applied. These data are, of course, excluded from the measurements provided here. The exact cause of the variation in the Pt sensor quality is unknown and being investigated.

\subsection{Electrochemical measurements}

CA and CV measurements were carried out using a Potentiostat ( $\mu$ autolab type II, Autolab) in combination with GPES software (version 4.9.007, EcoChemie B.V.) connected to the QCM system. A Cypress system EE009 No leak $\mathrm{Ag} / \mathrm{AgCl}$ (diameter: $2 \mathrm{~mm}$ ) electrode was used as a reference (RE) and a platinum plate functioned as the counter electrode (CE). The working electrode (WE) in this threeelectrode system was the $300 \mathrm{~nm}$ thick platinum film (electrode area: $0.79 \mathrm{~cm}^{2}$ ) on a disc-shaped piezoelectric crystal described above. A schematic illustrating the arrangement of the electrodes in the flow cell is shown in Figure 1. The CV sweeps were performed between $1.0 \mathrm{~V}$ and $1.3 \mathrm{~V} \mathrm{vs.} \mathrm{Ag} / \mathrm{AgCl}$ at the scan rates 1 and $5 \mathrm{mV} / \mathrm{s}$. Before each experimental run, the potential was held constant at $1.0 \mathrm{~V}$ for more than 5 minutes to allow oxidation of the Pt surface to come to equilibrium. Maintaining the potential above $1.0 \mathrm{~V}$ eliminates the reduction and reoxidation of Pt during the measurement. The open circuit potential (OCP) of the bare Pt electrode on the quartz crystals before application of any potential was $270+/-60 \mathrm{mV}$. 


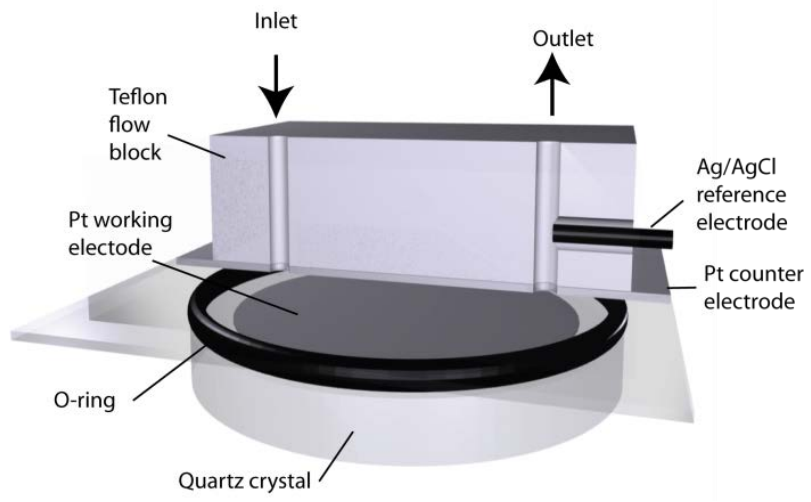

Figure 1. Schematic showing the relative positions of the quartz crystal, including the Pt working electrode, the $\mathrm{Ag} / \mathrm{AgCl}$ reference electrode, and the Pt counter electrode in the flow cell used. The front half of the Teflon flow block and Pt counter electrode have been rendered transparent to make the inside of the cell visible. (Not to scale.)

\section{Results and Discussion}

In application areas such as in the coating of electrodes for implantation, it is of importance to understand the adsorbing mechanism of the deposited material and, also, establish a better understanding of possible factors that may affect the quality of the resulting film. Here, we address the influence of the applied potential on the film growth process, and describe the results of varying the applied potential over time with a saw wave and in a step-wise fashion and the consequences these methods have on the experimental and on the resulting films.

\subsection{Influence of applied potential}

The applied potentials used to accelerate PLL deposition in this paper exceed $1 \mathrm{~V}(\mathrm{vs}$. $\mathrm{Ag} / \mathrm{AgCl})$ where significant oxidation of water begins (at $\mathrm{pH}$ around 7 and on $\mathrm{Pt}$ ). We therefore begin by taking a closer look at these processes. The applied potential required for an electrochemical process to occur is well depicted in a voltammogram, such as those shown in Figure 2a and d. Figure 2a-c present data from the 
$2^{\text {nd }}$ cycle of the HEPES CV experiment shown in Figure $3 v s$. the applied potential. Figure $2 a$ is the classic electrochemical voltammogram, showing the measured current vs. the applied potential. As expected for solvent electrolysis, the slope of the curve increases rapidly at higher potentials. During the return sweep, the measured current is slightly lower, likely because the electrolyte adjacent to the electrode surface has accumulated oxidation products $\left(e . g . \mathrm{H}^{+}\right.$and $\left.\mathrm{O}_{2}\right)$. Figure $2 \mathrm{~b}$ shows $\Delta \mathrm{f}_{5}$ over the same potential range. Here it becomes clear that oxidation of the solvent and/or removal of Pt affects the resonance frequency of the crystal at and above $1.2 \mathrm{~V}$. The decrease in $\Delta \mathrm{f}_{5}$ on the return sweep at potentials below $1.15 \mathrm{~V}$ appears to be the dissipation of dissolved $\mathrm{O}_{2}$ or $\mathrm{O}_{2}$ bubbles adjacent to the Pt sensor surface, while the net increase in $\Delta f_{5}$ of approximately $1.7 \mathrm{~Hz}$ suggests that $P t$ was removed or that one or more bubbles remained on the Pt surface. The removal of Pt is confirmed by the total change in the crystal's mass, discussed in section 3.4.2. The dissipation (shown in Figure 2c) shows no significant change until the reverse potential sweep (1.3 V-1.15 V), where $\Delta D_{5}$ increases until the entire cycle is nearly completed. In the end of the cycle $\Delta D_{5}$ decreases. Again, this is consistent with one or several bubbles at the Pt surface during this portion of the sweep.
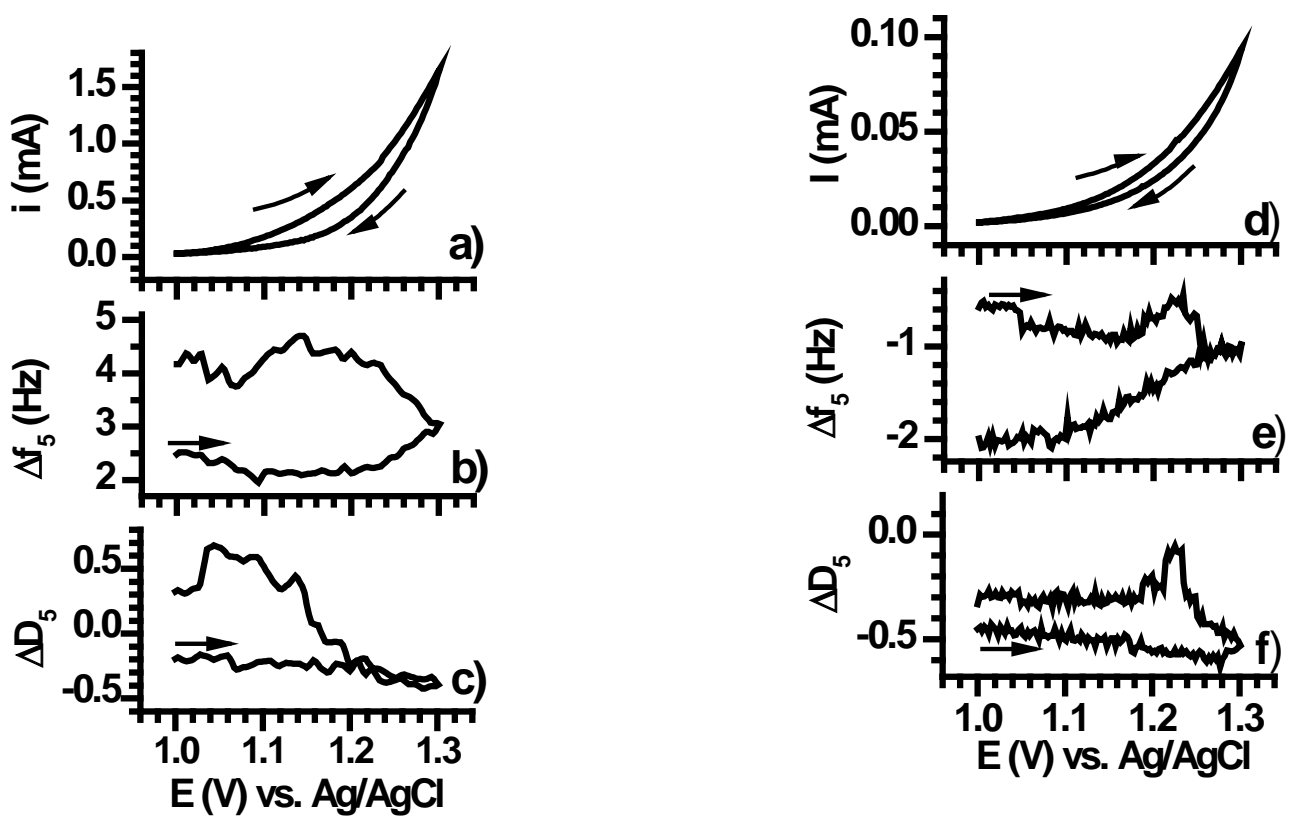

9 of 27 
Figure 2. $2^{\text {nd }}$ cycle of HEPES CV at a scan rate of $5 \mathrm{mV} / \mathrm{s}(\mathrm{a}-\mathrm{c})$ and $2^{\text {nd }}$ cycle of PLL adsorption at a scan rate of $1 \mathrm{mV} / \mathrm{s}(\mathrm{d}-\mathrm{f})$ on Pt WE up to $1.3 \mathrm{~V}$ vs. $\mathrm{Ag} / \mathrm{AgCl}$. (a,d) Current (I) vs. the potential (E). (b,e) The shift in resonance frequency $\Delta f_{5}$ and $(c, f)$ change in damping factor $\Delta D_{5}$.

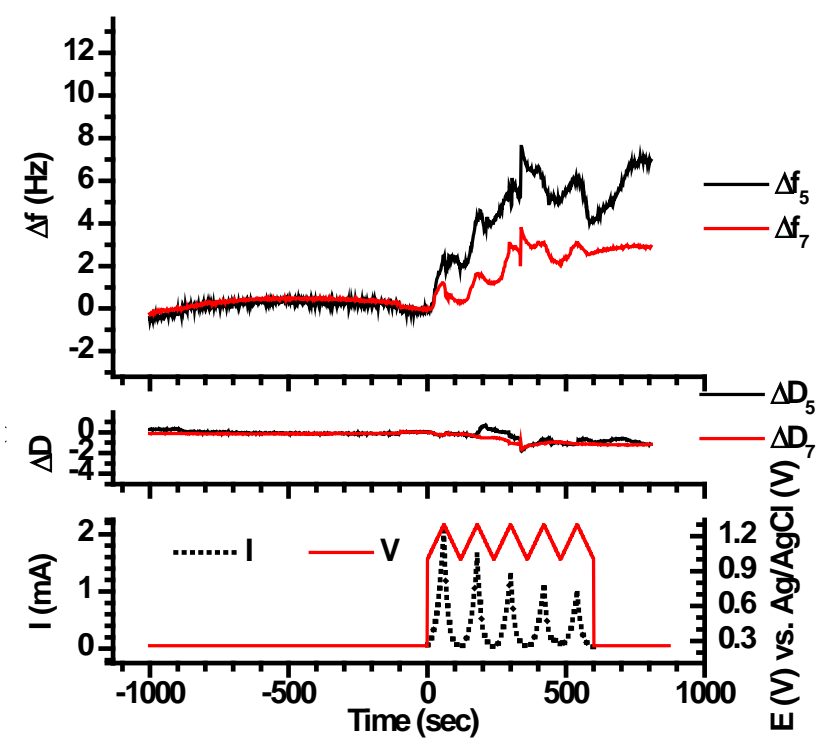

Figure 3. The shift in resonance frequency $\Delta f_{5}$ (top) change in damping factor $\Delta D_{5}$ (middle) and I and $E$ (bottom) vs. time for a HEPES CV experiment ( $\mathrm{NaCl}$ concentration $0.1 \mathrm{M}$ ) when cycling the potential between $1.0 \mathrm{~V}$ and $1.3 \mathrm{~V} v \mathrm{vs} . \mathrm{Ag} / \mathrm{AgCl}$ at $5 \mathrm{mV} / \mathrm{s}$. 


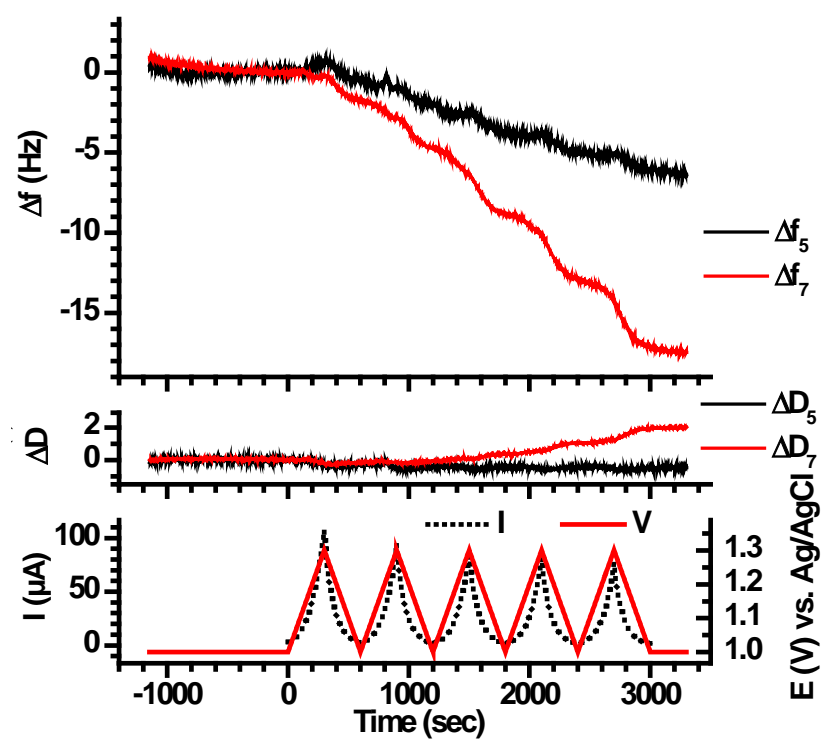

Figure 4. PLL adsorption studied via EQCM-D and CV, 5 cycles, varying the potential between $1.0 \mathrm{~V}$ and $1.3 \mathrm{~V}$ vs. $\mathrm{Ag} / \mathrm{AgCl}$ at a scan rate of $1 \mathrm{mV} / \mathrm{s}$.

Figure $2 \mathrm{~d}-\mathrm{f}$ show data analogous to that in Figure 2a-c for the HEPES solution containing PLL (full experiment shown in Figure 4). Here, the scan rate is decreased to $1 \mathrm{mV} / \mathrm{s}$ in order to follow the PLL deposition in detail. The shape of the voltammogram in Figure $2 \mathrm{~d}$ is very similar to that in Figure $2 \mathrm{a}$, although the magnitude of the current is significantly smaller. This is likely due to the interference of PLL on the Pt surface, decreasing the availability of Pt for oxidizing water and inhibiting transport of $\mathrm{O}_{2}$ and $\mathrm{H}^{+}$away from the surface, and the lower scan rate. $\Delta \mathrm{f}_{5}$ in Figure 2e shows that the deposition of PLL starts in earnest when the potential reaches its peak and continues through the rest of the cycle. The concomitant "bumps" in the $\Delta f_{5}$ and $\Delta D_{5}$ curves are probably the result of a bubble formed and released at around 1.15-1.25 V in the forward sweep. The resulting $\Delta \mathrm{f}_{5}$ ends up in a net deposition of approximately $1.5 \mathrm{~Hz}$. Note that this value does not include any correction for Pt that was likely lost during the same process (see the discussion in sections 3.4.2 and 3.5). 
The deposition of PLL, as inferred from Figure 2e, does not directly correlate with any discernible feature in the current curve (Figure $2 \mathrm{~d}$ ). The fact that deposition only appears to become significant after the electrolysis of water has also become significant, based on the measured current, suggests that the accelerated deposition of PLL may depend on one or more products from water electrolysis. However, as discussed by van Tassel et al. $[7,8]$, the local decrease in $\mathrm{pH}$ expected from the anodic electrolysis of water would be expected to increase coulombic interchain repulsion within the PLL, which can hardly be expected to accelerate the film deposition rate.

\subsection{Cyclic voltammetric PLL film growth}

In Figure 4, the frequency $\Delta \mathrm{f}$, dissipation $\Delta \mathrm{D}$, and current I measured as a function of time during a cyclic voltammetry experiment with PLL are shown. In the upper graph, one can follow the change in frequency, $\Delta f_{5}$ and $\Delta f_{7} v s$. time. PLL is deposited on the Pt-electrode without the disturbance of large $\mathrm{O}_{2}$-bubbles evolving at the liquid-surface interface. However, when reaching $1.3 \mathrm{~V}$ in the forward sweeps, $\Delta \mathrm{f}_{5}$ increases slightly (presumably $\mathrm{O}_{2}$-gas evolution) followed by a decrease due to the adsorption of PLL. The same trends are seen in $\Delta f_{7}$, but with a larger contribution of the adsorption of PLL, which in turn almost eliminates the $\mathrm{O}_{2}$-gas evolution signal. The $\Delta \mathrm{D}$ for both harmonics changes very little, and if anything increase slightly in a stable manner over time, suggesting that the film being deposited is plastic and/or viscoelastic.

The current I decreases in each CV cycle. This is expected, as the PLL hinders the transport of solvent to, and $\mathrm{O}_{2}$ and $\mathrm{H}^{+}$from, the anode. The magnitude of the change in frequency also increases with each cycle, indicating a small acceleration of the film deposition rate.

Figure 5 presents a longer CV experiment performed under similar conditions, but at a faster scan rate and during a longer period of time. During the first several hundred seconds, PLL was deposited relatively rapidly onto the Pt quartz crystal sensor surface, as visible in the change in $\Delta \mathrm{f}$. The relatively 
small increase in $\Delta \mathrm{D}$ during the same period suggests that the PLL film is relatively plastic/viscoelastic. After this initial period, the rate of change in $\Delta f$ decreases and $\Delta D$ begins to decrease. This can be explained by two likely mechanisms: 1) that the evolution of gas and the removal of Pt from the sensor surface balance the PLL deposition, or 2) a change in the film's internal molecular structure alters the film's rheology. The latter has been suggested to explain the change in deposition rate observed by others $[7,8]$. Whatever the mechanism, $\Delta$ f remains relatively constant until about $3000 \mathrm{~s}$ into the experiment. Between $4000 \mathrm{~s}$ and $9600 \mathrm{~s}$, the growth of the PLL film results in a drop in resonance frequency of 10 to $20 \mathrm{~Hz}$ (depending on the overtone). This process continued even after the applied potential was held constant at $1 \mathrm{~V}$ at the end of the experiment. The divergence between $\Delta f_{5}$ and $\Delta f_{7}$ (and between $\Delta D_{5}$ and $\Delta D_{7}$ ) in this region may indicate that the properties of the film vary with the distance from the electrode interface. The acoustic waves of lower frequency penetrate further into the film than those of the higher harmonics. In this case, the fact that $\Delta D_{5}$ and $\Delta f_{5}$ exhibit more dramatic changes than their $7^{\text {th }}$-harmonic counterparts suggests that the film closest to the electrode is relatively compact, rigid, and contains less water that the material further from the electrode.

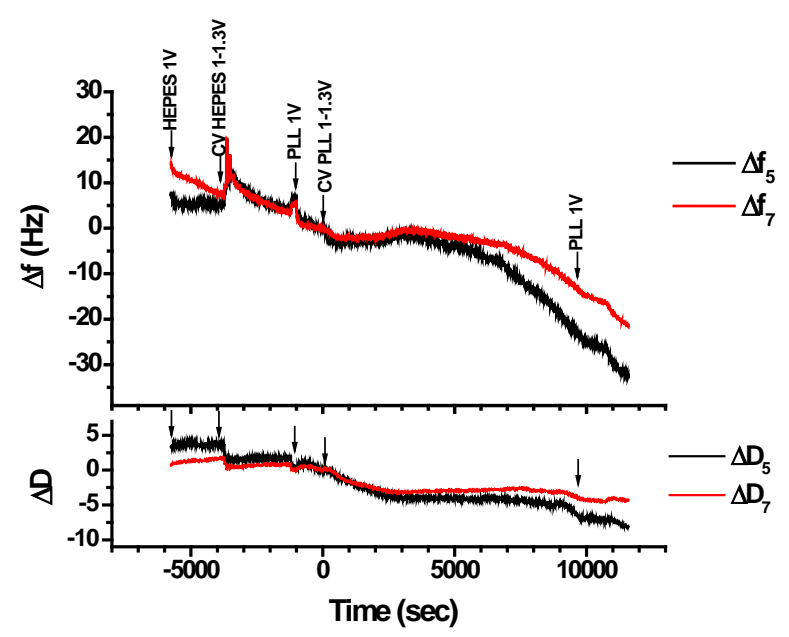


Figure 5. PLL adsorption studied via EQCM-D and CV, 80 cycles, varying the potential between $1.0 \mathrm{~V}$ and $1.3 \mathrm{~V} v s . \mathrm{Ag} / \mathrm{AgCl}$ at a scan rate of $5 \mathrm{mV} / \mathrm{s}$.

\subsection{Chronoamperometric PLL film growth}

Previous studies of PLL deposition onto anodically-addressed electrodes have primarily been performed at a constant potential $[7,8,16]$. Figure 6 shows the same parameters and observables as Figure 4 and Figure 5 for one such experiment where a potential of $1.3 \mathrm{~V}$ vs. $\mathrm{Ag} / \mathrm{AgCl}$ was applied for 600 $\mathrm{s}$ followed by $1 \mathrm{~V}$ for $600 \mathrm{~s}$ in a repeated fashion for a total series of 10 steps. While the $1.3 \mathrm{~V}$ potential is applied, the evolution of oxygen gas interferes with the direct measurement of the PLL film by the QCM. This is visible in a temporary increase in $\Delta \mathrm{f}$. By periodically decreasing the potential to $1 \mathrm{~V}$, we allowed the gas to disperse, so that a stable frequency (and dissipation) measurement reflecting the properties of the PLL film could be acquired.

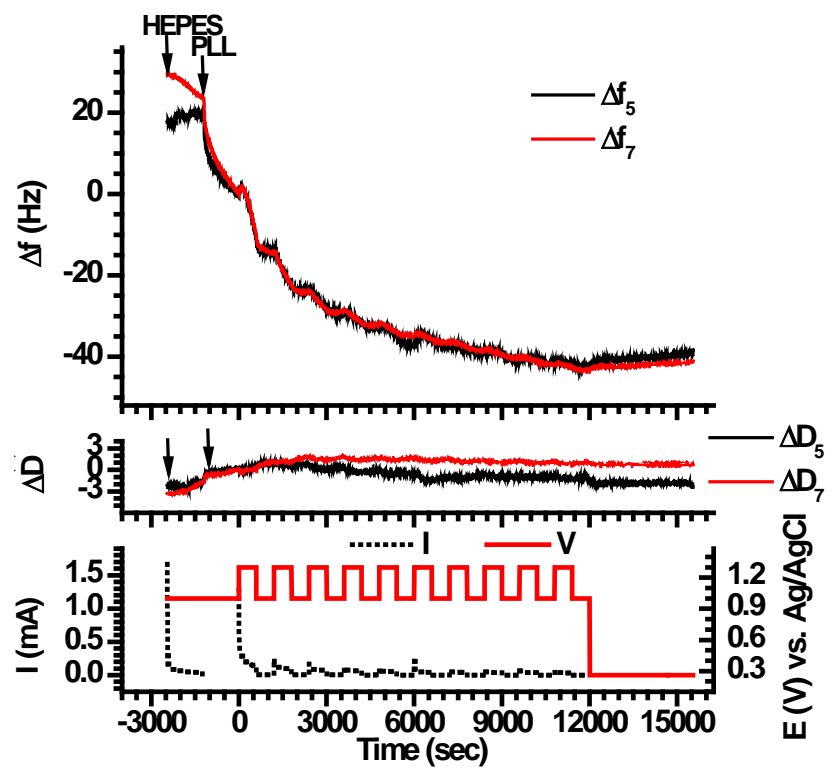


Figure 6. EQCM-D measurement of the adsorption of PLL $(0.4 \mathrm{~g} / \mathrm{I})$ in HEPES buffer on Pt. The applied potential was $1 \mathrm{~V}$ for $600 \mathrm{~s}$ followed by $1.3 \mathrm{~V}$ for $600 \mathrm{~s}$ ( $10 \mathrm{cycles}$ in total), vs. $\mathrm{Ag} / \mathrm{AgCl}$.

Notice that, as was the case with CV, the rapid deposition of PLL, indicated by the decrease in $\Delta \mathrm{f}$, slows, and after approximately $1000 \mathrm{~s}$, the dissipation within the film begins to decrease rather than increase. This behavior becomes even more evident in a plot of $\Delta \mathrm{D} v s$. $\Delta \mathrm{f}$, as shown in Figure 7 . For the initial phase of the deposition, $\Delta D_{5}$ increases as the film becomes thicker ( $\Delta f_{5}$ decreases), indicating that this portion of the film is relatively plastic or viscoelastic. The very rapid change in $\Delta f_{5}$ (best seen in Figure 6) also suggests that the deposition occurs quickly, or that the PLL is hydrated to a significant degree, adding to the effective mass measured by the crystal. Below about $-15 \mathrm{~Hz}$, the direction reverses, and the general trend shows that $\Delta D_{5}$ decreases while $\Delta f_{5}$ continues to decrease, indicating that the film is becoming much more rigid. This behavior is consistent with a change in the organization of the film from a relatively disordered structure, to a relatively ordered and rigid structure. See for example Zhang and $\mathrm{Wu}[23]$. 


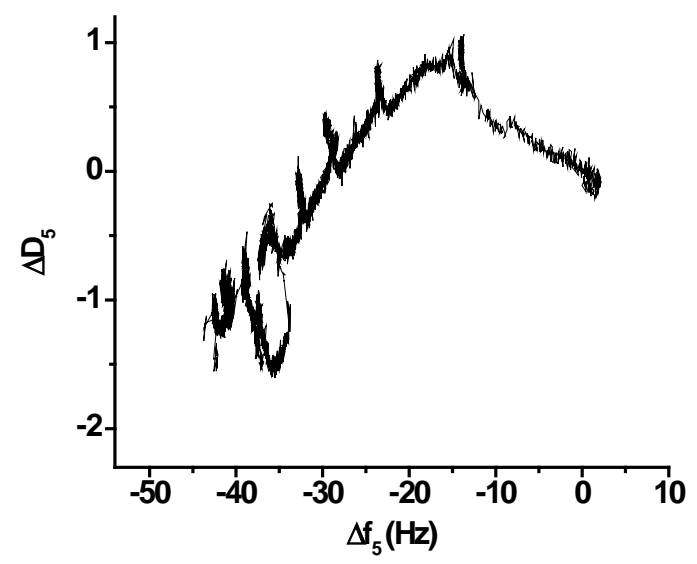

Figure 7. $\Delta \mathrm{D}$ versus $\Delta \mathrm{f}$ for overtone 5 from the same data shown in Figure 6 for the time period 0 to 12000 seconds.

Compared to the measurements reported by van Tassel et al. on ITO $[6,7,16]$, where the faradaic current density is reported to be approximately $600 \mathrm{nA} / \mathrm{cm}^{2}\left(200 \mathrm{nA} / 32 \mathrm{~mm}^{2}\right.$ surface area of the OW $2400 \mathrm{c}$ sensor) at $>1.3 \mathrm{~V}$ ( $1.5 \mathrm{~V}$ relative to the standard hydrogen electrode), the Pt surfaces used here appear to demonstrate significantly larger current densities at similar potentials, e.g. $65 \mu \mathrm{A} / \mathrm{cm}^{2}$ $\left(57 \mu \mathrm{A} / 0.88 \mathrm{~cm}^{2}\right)$ or 100 times the current density at $1.3 \mathrm{~V}$. It also appears that gas evolution occurs much more rapidly on Pt surfaces than on ITO, as van Tassel et al. [7] mention nothing about oxygen or gas production in their experiments, and even though the OWLS technique differs radically from QCM, we expect that the formation of gaseous bubbles on their electrodes would have been detected. This difference is not unexpected, as it is well-established that Pt is a better catalyst than ITO for the electrolytic oxidation of water. We present the characteristics and average growth rate of the PLL films below, after a discussion of the challenges caused by the (undesired) electrochemical reactions in the system.

\subsection{Electrochemical complications}


Our goal of studying the mechanism of PLL deposition onto Pt surfaces with EQCM-D was complicated by the formation of gas bubbles due to the electrolysis of water and the removal of Pt. In the coming sections we will demonstrate that the signals from the bubbles formed under the film due to electrolysis of the solvent easily can be distinguished from the adsorbed PLL by e.g. intermittently decreasing the applied potential (in a CV or pulsed CA experiment) during the deposition process.

\subsubsection{Oxidation (electrolysis) of water in HEPES}

As mentioned in the beginning of section 3, the applied potentials used are enough to oxidize water via the reaction:

$$
2 \mathrm{H}_{2} \mathrm{O} \rightarrow \mathrm{O}_{2}+4 \mathrm{H}^{+}+4 e^{-}
$$

The $\mathrm{O}_{2}$ produced either dissolves into the aqueous electrolyte or forms bubbles at the metal-liquid interface. The impact of these bubbles on the resonance frequency measured in the QCM is clearly visible in the upper graph in Figure 3, which shows a cyclic voltammogram from a measurement performed on a bare Pt QCM sensor in a HEPES electrolyte (without PLL). In this measurement, the applied voltage was cycled between $1.0 \mathrm{~V}$ and $1.3 \mathrm{~V}$ vs. $\mathrm{Ag} / \mathrm{AgCl}$. At $1.0 \mathrm{~V}$, the electrolysis of water is very small, as indicated by the current in the lower graph. When the applied potential exceeds about $1.2 \mathrm{~V}, \Delta \mathrm{f}$ (in both harmonics) increases sharply, and then decreases again as the applied voltage approaches its minimum. These events are most pronounced in the first 2-3 cycles, followed by a more complex/random, and potentially chaotic, behavior in the last cycles. The temporary increase in $\Delta f$ indicates a temporary decrease in the effective mass on the crystal. As with the PLL depositing measurements, we attribute this to the generation of $\mathrm{O}_{2}$ gas, which either forms small bubbles or a film at the interface between the Pt and the electrolyte, or is dissolved or entrained by the aqueous electrolyte near the interface, effectively decreasing the mass and viscosity of the HEPES solution locally. 
The net change in $\Delta f_{5}$ over the course of the experiment of about $4 \mathrm{~Hz}$ indicates either that bubbles accumulate at the Pt interface, or that Pt is removed from the crystal during the measurement. Based on the net change in the resonance frequency of the crystal in a dry environment (see section 3.4.2), measured before and after similar experiments, the removal of Pt appears to be the dominant effect.

Figure 8 shows an example of an experiment similar to that shown in Figure 6, in which one or more bubbles clearly form at approximately $10500 \mathrm{~s}$. The rapid and large change in both $\Delta \mathrm{f}$ and $\Delta \mathrm{D}$ are very different from the relatively gradual changes observed in Figure 6. The resonance frequencies and respective dissipation of the individual overtones of the quartz crystal respond at different times. This suggests that the disturbance from the bubble(s) was not evenly distributed over the crystal surface (see Rodahl et al. [24]). Both overtones react to another apparent bubble at about $12000 \mathrm{~s}$. Notice that the dissipation decreases when these bubbles form, since the viscous film and electrolyte no longer contact the entire Pt sensor surface. In this case, the bubbles effectively lubricate the surface, reducing the electrolyte's ability to dissipate the crystal's acoustic energy. Such large bubbles, formed under the PLL film, rarely leave the surface of the Pt sensor, but rather remain until the cell is opened. They can rupture the PLL film, leaving a visible defect in the resulting film, and decreasing the total mass of PLL deposited on the Pt surface. 


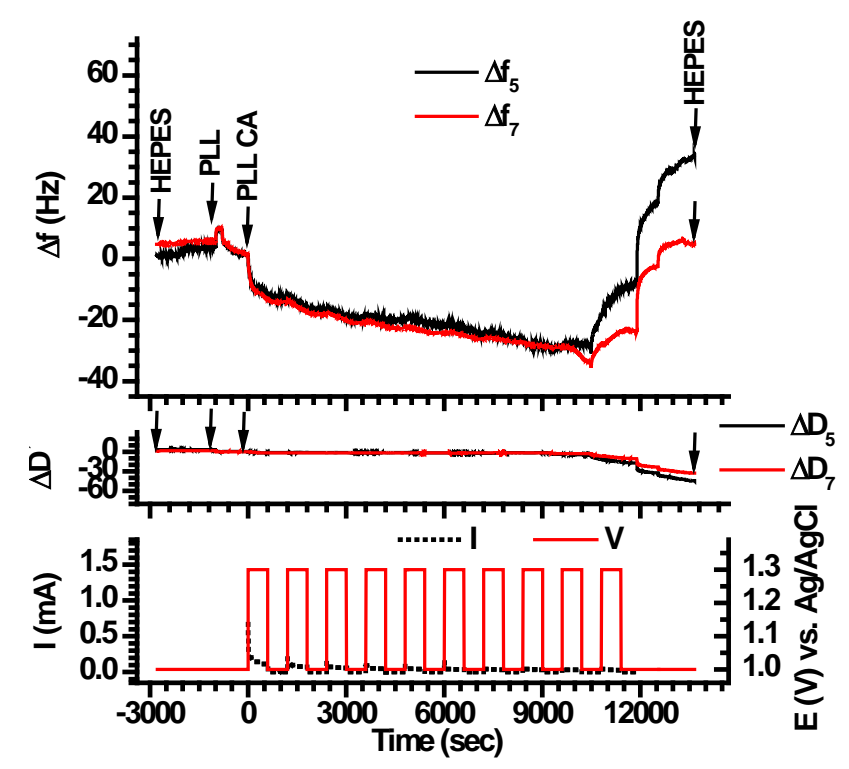

Figure 8. EQCM-D measurement of the adsorption of PLL $(0.4 \mathrm{~g} / \mathrm{l})$ in HEPES buffer on Pt. The applied potential was $1 \mathrm{~V}$ for $600 \mathrm{~s}$ followed by $1.3 \mathrm{~V}$ for $600 \mathrm{~s}$ (10 cycles in total), vs. $\mathrm{Ag} / \mathrm{AgCl}$ (the same conditions as in Figure 6. Here, one or more bubbles clearly form on the sensor surface (starting at around $10500 \mathrm{~s})$.

Figure 9 and Figure 10 show pictures of two Pt crystals after anodic PLL deposition. Figure 10 is an example where the EQCM-D data indicated the formation of bubbles during CV electrodeposition up to 1.3 V. We attribute the clearly-visible circular defects in the resulting PLL film to the edges of bubbles formed under the film by electrolytically-generated $\mathrm{O}_{2}$. As seen in the picture, the edges of the bubbles extend beyond the boundary of the Pt electrode to the bare quartz. Figure 9 shows a "smoother" PLLfilm for which the QCM traces indicated no large bubbles during electrodeposition under the same conditions. 


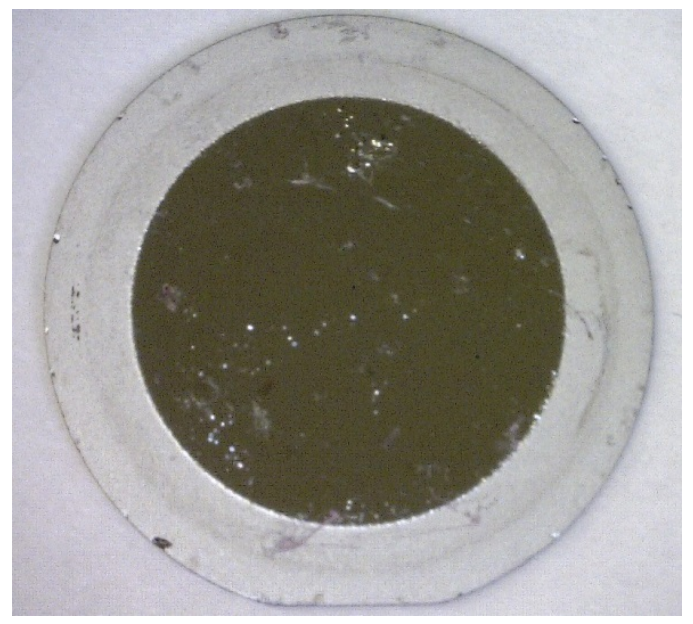

Figure 9. A Pt QCM sensor with an electrodeposited PLL film for which bubble formation was not indicated by the QCM data.

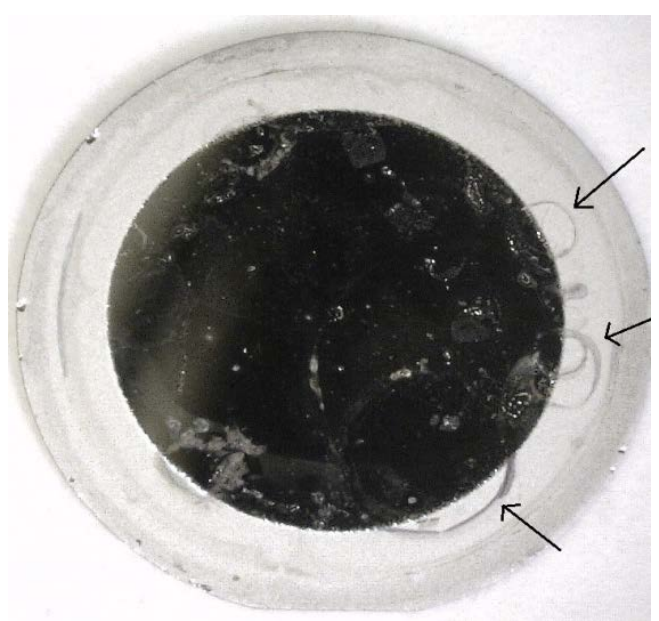

Figure 10. A Pt sensor surface with a PLL film for which the QCM indicated the formation of gas bubbles during electrodeposition. We attribute the large circular defects indicated by the arrows to electrolytically-generated $\mathrm{O}_{2}$ bubbles.

\subsubsection{Loss of Pt}

By measuring $\Delta \mathrm{f}_{5}$ on a dry crystal (in $\mathrm{N}_{2}$ ) before and after a measurement, one can calculate the total change in dry mass on the quartz sensor over the course of the deposition. This includes any PLL 
deposited on the surface of the Pt, but is also affected by any Pt lost during the experiment. Removing the PLL from the Pt surface (rinsing with Milli-Q followed by a TL-1 wash as described in section 2.1, exposing the Pt to air plasma and finally rinsing in Milli-Q water again before manually drying the Pt surface with $\mathrm{N}_{2}$ gas) and measuring the resonance frequency of the dry crystal again allows us to distinguish the mass of the PLL added and the Pt removed. Note that this required the removal and replacement of the crystal in the QCM module, which introduces a new uncertainty of a few $\mathrm{Hz}$ in the measurement of the absolute resonance frequencies used in this calculation. In general, the Pt etch rate was measured to be of the order of $0.5 \mathrm{ng} \mathrm{cm}^{-2} \mathrm{~min}^{-1}$.

The electrochemical etching of Pt has been reported previously [25], and in our experiments is likely accelerated by the $\mathrm{Cl}^{-}$in the electrolyte via the oxidation of $\mathrm{Pt}$ to $\mathrm{PtCl}_{4}$ or $\mathrm{PtCl}_{6}{ }^{2-}$. Although the $\mathrm{NaCl}$ $(0.1 \mathrm{M})$ we added to the HEPES buffer was not necessary to maintain the $\mathrm{pH}$ in the system, it is a physiologically relevant concentration for extracellular cell media [26], and has also been shown to increase the PLL deposition rate on Pt [7].

We also tested these processes up to an applied potential of $1.6 \mathrm{~V} v s$. $\mathrm{Ag} / \mathrm{AgCl}$. As expected, $\mathrm{Pt}$ etching and gas formation were both dramatically accelerated, as was the net deposition rate of PLL.

\subsection{Net growth rate of PLL film}

The formation of bubbles, without knowing their exact size or location, precludes the use of the sophisticated models available for extracting film properties (thickness, viscosity and/or mass) by analyzing QCM-D measurements. However, rough estimates can be made.

For $\mathrm{CV}$ and $\mathrm{CA}$ (with a $50 \%$ duty cycle) between $1 \mathrm{~V}$ and $1.3 \mathrm{~V}$ vs. $\mathrm{Ag} / \mathrm{AgCl}$ like the measurements shown in Figure 5 and Figure 6, the net PLL deposition rate, calculated from QCM measurements on dry films after the experiment, are very similar. CV results in a slightly higher average deposition rate of $8.8 \mathrm{ng} \mathrm{cm}^{-2} \mathrm{~min}^{-1}$, marginally larger than the $8.1 \mathrm{ng} \mathrm{cm}^{-2} \mathrm{~min}^{-1}$ we measure for CA. This would suggest 
that the enhanced PLL deposition occurs, on average, for more than half of each CV cycle. We also find that the Pt etch rate for CV is slightly lower than for CA, although it is not clear that this is statistically significant.

These electrodeposition rates are comparable to the rate reported for PLL on ITO under comparable conditions at a rate of $6 \mathrm{ng} \mathrm{cm}^{-2} \mathrm{~min}^{-1}$ [7]. This similarity is surprising considering the previouslymentioned difference in current density.

Preventing the formation of bubbles like those that destroy the film in Figure 8 is of obvious interest in applications where rapid uniform film growth is important (e.g. coating electrodes for implantation). Decreasing the applied potential, pausing frequently (e.g. pulsed CA or CV with a high sweep rate) and limiting the thickness of the film grown all decrease the risk of bubble formation and subsequent film rupture. These methods allow the gas and $\mathrm{H}^{+}$generated at the interface to dissipate into the electrolyte, rather than accumulate under and within the PLL film. However, they all limit the rate of film growth. This trade-off is unavoidable, as the rapid growth of the PLL film appears to require a potential above the oxidation potential of water, as can be observed in the $\Delta f_{5}$ curve in Figure $2 e$, and may depend on electrolytically-formed protons. However, the fact that we observe approximately the same PLL deposition rate on Pt as that observed by others on ITO [7], even though the observed current density is much higher for the Pt surfaces, suggests that it is not simply the protons that are accelerating the deposition process.

For comparison, we measured the deposition of PLL under similar conditions with no applied potential (Pt electrode at OCP). Here, we observed a decrease in $\Delta \mathrm{f}_{5}$ on the order of $10 \mathrm{~Hz}$ over two hours while $\Delta D_{5}$ increased by 2 units. The majority of the changes occurred in the first minutes of the measurement, caused by the difference in viscosity and density between the PLL solution and the HEPES buffer. As such, very little PLL was deposited on the Pt electrode without the application of an anodic potential. 


\subsection{Characteristics of PLL films}

As commented by van Tassel et al., the fact that the charge on the PLL chains in solution is largely positive at $\mathrm{pH}<7$, as would be the case near the surface of the QCM crystal during the anodic electrolysis of water, would suggest that the film growth rate should be suppressed [7, 15]. Observations that the film growth rate increases with applied potential suggests another mechanism must come into play, overcoming the electrostatic repulsion of the charged polymer segments. This may be facilitated by anions from the buffered electrolyte (see [ $\mathrm{NaCl}]$ dependence reported in) [7], which are expected to be found in high concentration at and near the interface between the electrolyte and anode. However, van Tassel [8] also hypothesized that, at low pH, the long-term, constant rate growth of the film could represent a transition to a structure with the PLL molecules oriented orthogonally to, rather than parallel to, the electrode surface. We observe a change in the deposition rate after approximately 1000 to 3000 seconds of deposition, depending on the conditions, consistent with the change observed by his group [6, 7]. More importantly, the change in dissipation observed with the QCM after similar deposition times in our experiments indicates that the viscoelastic properties of the film are changing from relatively viscous to more rigid. This, at first glance, would seem to contradict the hypothesis presented by van Tassel [8], in which the density of the film being deposited is predicted to decrease over time to increase the distance between the charged polymer segments. Instead, it would appear that another structure, potentially involving the sharing of anions between adjacent polymer chains, and hence increasing the mechanical connectivity between the chains, is involved. Such a mechanism would result in a more structured and rigid film, as observed in the $\Delta D$ measurements presented in Figure 5 and Figure 7.

If the structure of the polymer chains in the film depends on the potential applied, local $\mathrm{pH}$, or other conditions, it is feasible that part of the changes observed in Figure 5 and Figure 7 are simply the 
reaction of the film and electrolyte to the changing potential. To characterize this, Figure 11 and Figure 12 below show the CV of HEPES on at Pt crystal before (Figure 11) and after (Figure 12) the PLL deposition shown in Figure 5. The first shows the effect of oxidation of the electrolyte on the QCM crystal. A regularly repeating and temporary change in $\Delta f_{5}$ shows the response of the electrolyte, likely electrolytic gas evolution, at the surface in both graphs. However, after the PLL deposition, as shown Figure $10 \Delta D_{5}$ shows a clear decreasing trend over the course of the entire experiment. This would indicate that the film continues to become more rigid, and presumably more structured, under these conditions, even though PLL is no longer available for deposition from the electrolyte.

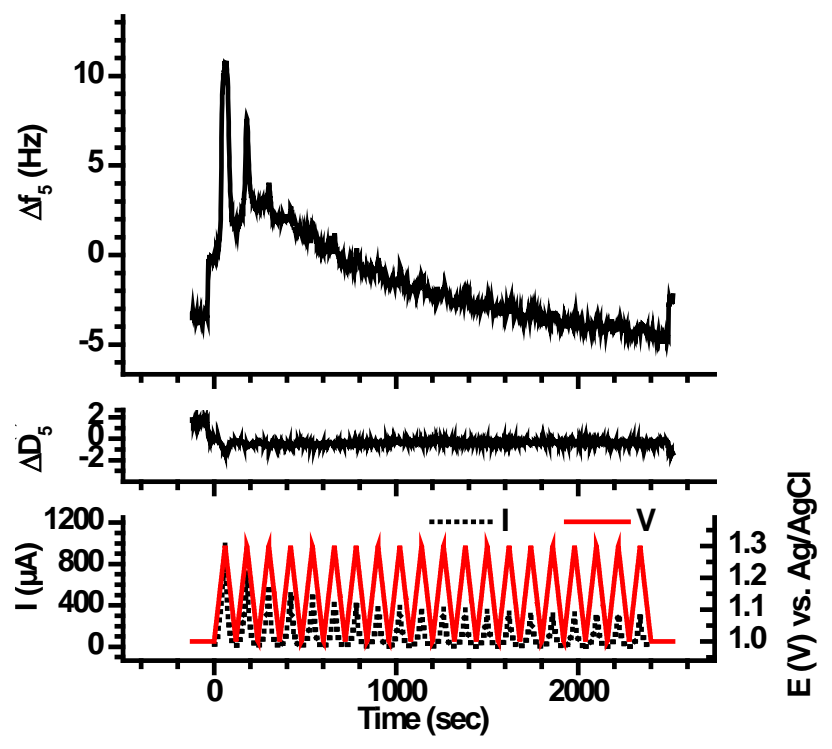

Figure 11. CV of HEPES ( $\mathrm{NaCl}$ concentration $0.1 \mathrm{M}$ ) when cycling the potential between $1.0 \mathrm{~V}$ and $1.3 \mathrm{~V}$ vs. $\mathrm{Ag} / \mathrm{AgCl}$ at $5 \mathrm{mV} / \mathrm{s}$ before $\mathrm{CV}$ deposition of PLL shown in Figure 5. 


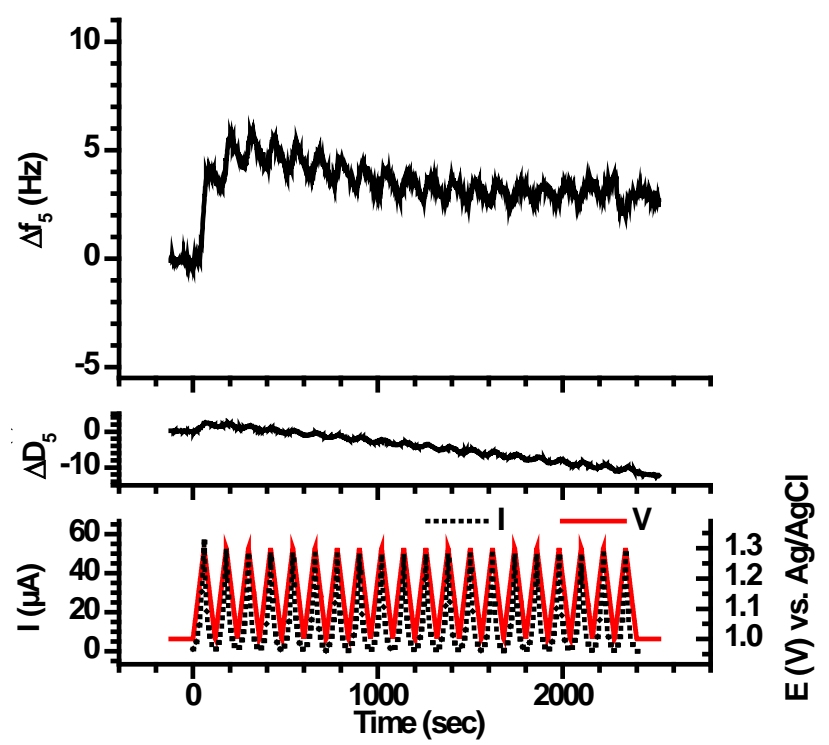

Figure 12. $\mathrm{CV}$ of HEPES ( $\mathrm{NaCl}$ concentration $0.1 \mathrm{M}$ ) when cycling the potential between $1.0 \mathrm{~V}$ and $1.3 \mathrm{~V}$ vs. $\mathrm{Ag} / \mathrm{AgCl}$ at $5 \mathrm{mV} / \mathrm{s}$ after $\mathrm{CV}$ deposition of PLL shown in Figure 5.

\section{Conclusion}

Rapid anodic electrodeposition of PLL onto Pt can be performed in a manner similar to that previously reported on ITO, and appears to follow a similar mechanism, resulting in a change in deposition rate and film properties after the first $600-3000$ seconds. We observe approximately $100 x$ the rate of water electrolysis on Pt compared to previous reports on ITO, but record similar PLL growth rates, suggesting that the local $\mathrm{pH}$ is not a dominating factor in the film growth process. The dissipation measured with the QCM indicates that the mechanical properties of the preliminary film are relatively viscoelastic or plastic. This changes concurrently with the change in film growth rate, and subsequent deposition results in a more rigid, and presumably compact, film. Furthermore, on $\mathrm{Pt}$, gas production is more apparent, and complicates the use of QCM as a measurement technique. However, pausing the electrochemical process (pulsed CA) or using CV allows the film and electrolyte near the quartz sensor to 
recover, resulting in frequency and dissipation data that can be interpreted. The electrochemical oxidation of $\mathrm{Pt}$ (apparently to $\mathrm{PtCl}_{4}$ ) also etches the Pt electrode under conditions required for rapid deposition of PLL. Fortunately, the various mechanisms can be (largely) deconvoluted by comparing the resonance frequencies (at several harmonics) and dissipation of wet and dry QCM sensors before and after film growth, and again after the deposited PLL film has been removed.

\section{Acknowledgements}

The authors wish to thank the reviewers who examined an early draft of this manuscript for their helpful insights and suggestions. NDR and SN thank the Swedish Research Council (Vetenskapsrådet) for grants 325-2008-7537 and 621-2007-3983 and Bo Liedberg and the Molecular Physics Group at Linköping University for access to equipment. FB acknowledges The Carl Trygger Foundation and The Swedish Foundation for Strategic Research for financial support.

The authors declare no financial conflict-of-interest with regards to the contents of this article.

\section{References}

[1] A. Mercanzini, K. Cheung, D.L. Buhl, M. Boers, A. Maillard, P. Colin, J.C. Bensadoun, A. Bertsch, P. Renaud, Sens Actuator A-Phys 143 (2008) 90-96.

[2] J.C. Williams, R.L. Rennaker, D.R. Kipke, Brain Research Protocols 4 (1999) 303-313.

[3] F. Boulmedais, C.S. Tang, B. Keller, J. Voros, Adv Func Mater 16 (2006) 63-70.

[4] M. Kawaguchi, A. Takahashi, Adv Colloid Interface Sci 37 (1992) 219-317.

[5] K.E. Bremmell, G.J. Jameson, S. Biggs, Colloid Surface A 139 (1998) 199-211.

[6] A.P. Ngankam, P.R. van Tassel, Langmuir 21 (2005) 5865-5871.

[7] A.P. Ngankam, P.R. van Tassel, P Natl Acad Sci USA 104 (2007) 1140-1145.

[8] P.R. Van Tassel, Current Opin Colloid In 17 (2012) 106-113.

[9] M.A. Cohen Stuart, C.W. Hoogendam, A. De Keizer, J Phys-Condens Mat 9 (1997) 7767-7783.

[10] F. Greveb, S. Frerkera, A.G. Bittermannc, C. Burkhardtd, A. Hierlemannb, H. Halla, Biomater 28 (2007) 5246-5258.

[11] O. Guillaume-Gentil, Y. Akiyama, M. Schuler, C. Tang, M. Textor, M. Yamato, T. Okano, J. Voros, Adv Mater 20 (2008) 560-565.

[12] L. Dieguez, N. Darwish, N. Graf, J. Voros, T. Zambelli, Soft Matter 5 (2009) 2415-2421.

[13] K. Sato, D. Kodama, Y. Naka, J. Anzai, Biomacromol 7 (2006) 3302-3305.

[14] J.P. Bearinger, J. Voros, J.A. Hubbell, M. Textor, Biotechnol Bioeng 82 (2003) 465-473. 
[15] Y.H. Ko, Y.H. Kim, J. Park, K.T. Nam, J.H. Park, P.J. Yoo, Macromolecules 44 (2011) 2866-2872.

[16] C. Olsen, P.R. van Tassel, J Colloid Interf Sci 329 (2009) 222-227.

[17] K.M. Gray, B.D. Liba, Y. Wang, Y. Cheng, G.W. Rubloff, W.E. Bentley, A. Montembault, I. Royaud, L. David, G.F. Payne, Biomacromolecules 13 (2012) 1181-1189.

[18] M. Rodahl, F. Hook, A. Krozer, P. Brzezinski, B. Kasemo, Rev Sci Instrum 66 (1995) 3924-3930.

[19] D.A. Buttry, M.D. Ward, Chem Rev 92 (1992) 1355-1379.

[20] G. Sauerbrey, Z Phys A-Hadron Nucl 155 (1959) 206-222.

[21] M.V. Voinova, M. Rodahl, M. Jonson, B. Kasemo, Phys Scr 59 (1999) 391-396.

[22] Q-Sense E4 Operation, Rev A Q-Sense AB, 2005.

[23] G. Zhang, C. Wu, Macromol Rapid Comm 30 (2009) 328-335.

[24] M. Rodahl, B. Kasemo, Sensor Actuat B-Chem 37 (1996) 111-116.

[25] A. Gencoglu, A. Minerick, Lab Chip 9 (2009) 1866-1873.

[26] D.M. Bers, W.H. Barry, S. Despa, Cardiovasc Res 57 (2003) 897-912. 